\title{
Who is Who in Carbon Balance and Management 2006 Georgii A Alexandrov*
}

\author{
Address: Institute of Atmospheric Physics, Russian Academy of Sciences, Pyzhevsky 3, Moscow, Russia \\ Email: Georgii A Alexandrov* - a_georgii@hotmail.com \\ * Corresponding author
}

Published: 22 January 2007

Carbon Balance and Management 2007, 2:1 doi:10.1186/1750-0680-2-1

This article is available from: http://www.cbmjournal.com/content/2/I/I

(C) 2007 Alexandrov; licensee BioMed Central Ltd.

This is an Open Access article distributed under the terms of the Creative Commons Attribution License (http://creativecommons.org/licenses/by/2.0), which permits unrestricted use, distribution, and reproduction in any medium, provided the original work is properly cited.
Received: 10 January 2007

Accepted: 22 January 2007

\begin{abstract}
This editorial provides a subject index from published articles, active researchers, and published papers in the field of carbon balance and management.
\end{abstract}

\section{Background}

Developing a policy relevant understanding of the global carbon cycle requires a high degree of interdisciplinarity. Therefore, research published in Carbon Balance and Management involves cutting across disciplines and consulting with specialists in various fields. This editorial is to support an interdisciplinary effort with relevant bibliographic information [see Additional file 1] including a source of names to consult with and a list of subjects that may appear in the papers. Terms may differ depending on context.

\section{Subject index}

This list of words and wordings [see Additional file 2], selected from the articles published in the first volume of the journal, represents the phraseology of carbon cycle science. It was compiled to provide help in searching relevant web resources. This list is also presented below together with links to the articles where a given subject is treated or mentioned.

ability to retain organic carbon [14]

acceptable climate change [6]

acid neutralizing capacity of seawater [4]

acid-base balance [2] acid-base imbalances in marine organisms [2]

activity data [9]

actual country-specific information [9]

adverse conditions [6]

afforestation $[15,134]$

agriculture sector [9]

alkalinity [2]

allocation of carbon [6]

anomalies in atmospheric $\mathrm{CO}_{2}$ increase [7]

anomalous $\mathrm{CO}_{2}$ flux [7]

anomalously extreme climate [6]

Anthropocene $[3,4]$

anthropogenic emissions [7]

Asia region [9] 
atmosphere-ice-ocean carbon cycle model [2]

atmospheric $\mathrm{CO}_{2}$ concentrations $[2,5,88]$

average surface temperature [2]

baseline energy consumption from air-conditioning [12]

baseline value for SOC [14]

biologically mediated $\mathrm{pH}$ changes [2]

biosphere simulation model [6]

burnt biomass [7]

calcareous shells [2]

capacity to absorb anthropogenic $\mathrm{CO}_{2}[5]$

capacity to act as sinks [3]

carbon budgets $[7,11,85,133]$

carbon conserving practices [4]

carbon cycle feedbacks $[3,42,44,49,50,81]$

carbon emissions $[8,11]$

carbon fertilisation $[6,53,55]$

carbon flows [8]

carbon flux anomalies [7]

carbon fluxes $[3,7,8,17,46,55,65]$

Carbon Management Education [13]

carbon price incentive schemes [15]

carbon sequestration $[4,15,107,134,135]$

carbon sink $[5,45,73]$

carbon stocks in forest biomass [15]

carbon tax [15]

carbon uptake [5-7, 15,36,49]

carelessness feedback [4]

cation exchange capacity [14] change of vegetation type [6]

changes in the moisture regime [3]

changes in weather patterns [6]

Cities for Climate Change Program [11]

climate change

$[2,6,14,18,40,43,44,46,49,58,60,81,108,134,136]$

climate change feedbacks on the carbon chemistry [2]

climate policy [5]

climate scenario [6]

$\mathrm{CO}_{2}$ biological pump [2]

$\mathrm{CO}_{2}$ emissions in the commercial sector [12]

$\mathrm{CO}_{2}$ uptake by the ocean $[2,34]$

collapse of the Amazonian rain forest [6]

compensating effects $[2,3]$

compensatory mechanisms [6]

consumption activities [9]

conversion of natural lands [8]

country-specific emission factors [9]

coupled atmosphere-ocean mode [2]

cover fraction of major vegetation types [6]

current forest cover [15]

data set $[7,8,34,63,133]$

dead organic matter [8]

decline in boreal forest area [6]

decline in forest area [6]

decrease in $\mathrm{pH}$ due to ocean warming [2]

decrease of biomass [6]

decreases in transpiration [6] 
decreasing rainfall [6]

default activity data [9]

default emission factors [9]

deforestation $[6,15,134]$

deforestation emissions [15]

deforestation tax [15]

desertification [14]

DIC concentrations [2]

direct anthropogenic emission of $\mathrm{CO}_{2}[8]$

direct effects of ocean warming [2]

direct human influence [5]

direct injection of carbon into the deep ocean [4]

dissolution of exoskeletal components [2]

disturbance in terrestrial ecosystems [3]

disturbances of the global carbon cycle [7]

drought-induced tree line [6]

Dynamic Global Vegetation Models $[6,43,48,61,70]$

dynamics of terrestrial ecosystems [3]

ecological modernization [11]

ecosystem physiology [3]

eddy covariance measurements [7]

EEZ [5]

EEZ carbon sink [5]

effect of income [11]

effects of temperature and precipitation [7]

embedded carbon [11]

emission factors $[9,12]$

emission reduction targets [12] emission sources $[9,105,106]$

emissions reduction target [11]

emitting mechanisms from sources [9]

energy consumption in typical offices [12]

energy savings potential [12]

energy usage within buildings [12]

energy use [11]

enhanced litter production [6]

enhanced plant growth [3]

environmental regulations [11]

environmental stress [6]

estimation methods [9]

exceptionally dry years [6]

Exclusive Economic Zone [5]

expanding land use [6]

extended dry seasons [7]

extent of ocean acidification [2]

feedbacks and non-linearities [8]

feedbacks and nonlinearities [3]

feedbacks between climate and vegetation [6]

financial mechanisms [15]

fire control in forests [4]

forest $[3,6,14,15,17,51,54,55,57,75,79,119,133]$

forest degradation [15]

forest expansion [15]

fossil fuel consumption [4]

fuelwood production [15]

full carbon budgets for cities [11] 
functional strategies [6]

geoengineering strategies [4]

geographical pattern of vegetation [6]

geologic sequestration [4]

GHG inventories [9]

global carbon trading [5]

global decline in SOC [14]

greenhouse gas emission scenario [6]

greenhouse gas emissions [11]

greenhouse gas inventories $[9,12]$

guidelines of the Intergovernmental Panel on Climate Change [9]

heat stress on boreal trees [6]

heating, ventilating, and air conditioning [12]

heterotrophic respiration [7]

holistic view of the carbon cycle [3]

human emissions-atmospheric $\mathrm{CO}_{2}$ equation [3]

human well-being [6]

HVAC [12]

impact of climate change $[6,14]$

incentives for keeping the forest carbon stock intact [15]

increase in fire frequency [6]

increase in oceanic [2]

increased deciduousness [6]

increased water demand [6]

increased water use efficiency [6]

increases in growing stock [15]

increasing concentration of carbonic acid [3] increasing greenhouse gas concentrations [6]

industrial carbon dioxide emissions [11]

industrialization [11]

information exchange activities [9]

injected carbon [4]

insensitivity of $\mathrm{pH}$ to climate change [2]

institutional drivers [11]

institutional impacts [11]

intergenerational equity [4]

invariant allocation of carbon gains [6]

IPAT $[11,104]$

IPCC guidelines [9]

Kuznets curve [11]

Kyoto Protocol $[4,5,134]$

land ecosystems [6]

land use change [7]

land use model [15]

Land Use, Land-Use Change and Forestry [9]

land-use change [11]

large-scale climate anomalies [7]

leaf nitrogen contents [6]

living biomass [8]

locally appropriate levers for carbon management [13]

locally derived regulatory oversight [11]

long term means to store $\mathrm{CO}_{2}[4]$

lower wet season precipitation [7]

magnitude of carbon fertilisation effects [6]

marine carbon cycle [3] 
marine organisms [2]

meridional change in $\mathrm{pH}[2]$

Miami model [14]

mitigating the heat load of buildings [12]

mitigation and adaptation policies [6]

mobilisation of nitrogen [3]

modernization effect [11]

Montreal Protocol [9]

national carbon accounts [5]

national communications [9]

national greenhouse gas inventories [11]

NEE anomalies [7]

net carbon exchange [6]

net carbon loss [6]

net climate change feedback [2]

net ecosystem production $[15,133]$

net loss of carbon [3]

net primary production $[7,14,71,72]$

net primary productivity $[71,116]$

net sink of carbon [6]

neutralizing capacity of calcium carbonate [4]

non-woody vegetation [6]

northward expansion of the boreal forest [6]

NPP $[7,8,14]$

nutrient and water constraints [6]

nutrient limitations of vegetation growth [6]

ocean acidification $[2,19]$

ocean $\mathrm{pH}[2,20]$ ocean warming [2]

ocean warming feedback [2]

oceanic anthropogenic $\mathrm{CO}_{2} \operatorname{sink}[5]$

oceanic $\mathrm{CO}_{2}$ uptake $[2,18,67]$

oceanic uptake of anthropogenic $\mathrm{CO}_{2}[2]$

past changes in climate [6]

permafrost soils [3]

permafrost thawing depth [6]

pest outbreaks [3]

$\mathrm{pH}$ of seawater [2]

phosphorus availability [14]

pools of carbon [3]

population growth $[11,92]$

purposeful carbon sequestration [4]

purposeful sequestration [4]

quantifiable mitigation strategies [14]

rate of acidification [2]

rates of leakage [4]

recession of the boreal forest [6]

reduce energy consumption in the HVAC [12]

reduced precipitation [6]

reduced soil respiration [7]

reducing deforestation [15]

reduction in DIC growth [2]

regional carbon budget [11]

regional changes in vegetation structure [6]

reporting country inventory practices [9]

reporting requirements [9] 
reservoir to purposefully sequester carbon [4]

response of marine organisms to $\mathrm{pH}$ changes [2]

response of vegetation [7]

responses of plant functional types to climate [6]

responses to a warming climate [3]

retention of forests [15]

rising acidity of the ocean [3]

robust assessment [6]

salinity [2]

saturation state of calcium carbonate [3]

sea-ice extent [2]

sectoral groups of energy [9]

sensitivity of future oceanic acidification [2]

sensitivity of heterotrophic respiration to soil moisture [7]

sensitivity to natural disturbances [6]

sequestration efficiency [4]

shifts in spatial rainfall distribution [7]

short-lived plant parts [6]

slow down climate change [6]

SOC [14]

socioeconomic drivers [6]

soil crusting and compaction [14]

soil microbial activity [7]

soil organic carbon $[14,108,109,112,115,130]$

soil organic matter $[7,14,112,114]$

soil processes [7]

soil respiration $[3,6,7,16,84]$

soil survey [14] solubility induced changes [2]

spatial distribution of vegetation [6]

speciation of carbon within the ocean [2]

species composition [6]

steady state analysis [14]

technological efficiency [11]

temporal development of vegetation [6]

temporal variability of heterotrophic respiration [7]

terrestrial balances of carbon [6]

terrestrial carbon balance [6]

terrestrial carbon cycling [14]

terrestrial carbon sink [6]

terrestrial feedback on climate [6]

terrestrial modulation of atmospheric carbon anomalies [7]

terrestrial vegetation $[6,52]$

the impact on soil respiration [3]

the lack of actual country-specific information [9]

the value of forest land [15]

thermodynamic equilibration [2]

transfer of $\mathrm{CO}_{2}$ from the atmosphere to the oceans [4]

transition from temperate savannah to subtropical woodland [6]

trophic structure of marine ecosystems [3]

uncertainty of estimated emissions [9]

UNFCCC [9]

UNFCCC requirements [9]

Urban and Regional Carbon Management [13]

urban areas [11] 
urban territories $[8,102]$

urban vegetation [8]

urbanised ecosystem [8]

urbanized areas [11]

value of the regional network [9]

variations in the $\mathrm{CO}_{2}$ growth rate [7]

voluntary environmental management standard [11]

vulnerable to rising temperatures [3]

water-limitation of NPP [7]

weakening oceanic sink [3]

WGIA [9]

wildfires [3]

woody encroachment [6]

world-wide cooling [7]

\section{Author index}

The list of names cited in the first volume of the journal provides some information about the research community involved in the study of the global carbon cycle either directly or indirectly. This information is intended for those who are considering Carbon Balance and Management as a medium for conveying their findings and evaluating whether they would be of sufficiently immediate interest to researchers in the broad range of disciplines associated with the studies of the global carbon cycle.

Acock, B. [128]

Adams, A.F.R. [112]

Adams, D.E. [131]

Aerts, K. [25]

Alcamo, J. [41]

Alder, J. [41]

Alexandrov, G. [135]

Alexandrov, G.A. $[133,10]$
Amato, M. [123,132]

Anderson, B. [80]

Andren, O. [122]

Angert, A. [74]

Apps, M.J. [17]

Arellano, A. [66]

Arneth, A. [70]

Asner, G.P. [71]

Asshoff, R. [55]

Aumont, O. [19]

Aumont, O.L. [67]

Bala, G. [50]

Baldocchi, D.D. [75]

Banks, H.T. [64]

Barrett, D.J. [116]

Barry, J.P. [24]

Bazilevich, N.I. [89]

Beaufort, L. [25]

Benitez, P.C. $[134,135]$

Benthien, A. [25]

Berry, J.A. $[83,82]$

Berthelot, M. [46]

Betts, R. [50]

Betts, R.A. $[57,42,51,43]$

Bignucolo, O. [55]

Biraud, S. [74]

Birdsey, R. [71]

Boden, T.A. [75] 
Bondeau, A. $[43,70]$

Bonfils, C. [74]

Bopp, L. [50,67,19]

Bousquet, P. [67,76,65]

Boutin, J. [36]

Brewer, P.G. [24]

Brovkin, V. $[43,50]$

Buck, K.R. [24]

Buermann, W. $[74,80,76]$

Bullister, J.L. [34]

Cadule, P. [50]

Caldeira, K. [20]

Campbell, J. [16]

Canadell, J. [129]

Canan, P. [13]

Catton, W.R. [91]

Chou, L. [25]

Ciais, P. $[46,67,65]$

Coleman, K. [120]

Collatz, G. [66]

Collatz, G.J. [83,77]

Collins, M. [51]

Colunga-Garcia, M. [108]

Cooper, C. [64]

Cornelissen, J.H.C. [16]

Cox, P. [50]

Cox, P.M. $[57,42,51,43,44]$

Cramer, J.C. [92]
Cramer, W. [41,43,56,76,6,47,70]

Crill, P. [79]

Cure, J.D. [128]

da Rocha, H.R. [79]

Dala, O.E. [129]

Dale, V.H. [110]

Daube, B.C. [79]

Davidson, E.A. [81]

Davis, M. [78]

de Camargo, P.B. [79]

de Freitas, H.C. [79]

DeAngelis, D.L. [110]

DeFries, R. [86]

DeFries, R.S. [77]

Delille, B. [25]

Diamond, L. [94]

Dickinson, R.E. $[80,1]$

Dickson, A.G. [39]

Dietz, T. [95,104]

Dix, M.R. [31]

Doney, S. [50]

Doney, S.C. [19]

Drapek, R.J. [59]

Dufresne, J.L. [46,44]

Duncan, O.D. [96]

Dunlap, R.E. [91]

Eby, M. [50]

Ehrlich, P. [98] 
Eichhout, B. [60]

Ellsworth, D.S. [54]

Emanuel, W.R. [110]

Engel, A. [25]

Erbrecht, T. [7,6]

Erlinger, J.R. [129]

Ewert, F. [118]

Fabry, V.J. [19]

Farquhar, G.D. [82,88]

Fasham, M.J.R. [88]

Feely, R.A. [19]

Field, C. [71]

Fisher, V. [43]

Foley, J.A. $[43,85,136]$

Francey, R. [67]

Friedlingstein, P. [46,44,50,76,65]

Friend, A.D. [43]

Fung, I. [74,50]

Gage, S.H. [109,108]

Garcia, H.E. [35]

Gattuso, J.P. [25]

GCTE, N.E.W.S. [16]

Genovese, V. [73]

Gerber, S. [61,49]

Gerten, D. [52,56,47,87]

Gifford, R.M. [117]

Giglio, L. [77,66]

Gnanadesikan, A. [19]
Gordon, C. [64]

Gordon, H.B. [30]

Goulden, M.L. [88,79]

Grace, P.R. [109,14,108,132]

Grant, R.F. [125]

Gregory, J.M. [64]

Gruber, N. [36,19,65]

Gu, L. [75]

Gurevitch, J. [16]

Haberlandt, U. [52]

Hanaoka, T. [106]

Hansen, J. [68]

Hansen, M. [86]

Hansen, P.J. [27, 28]

Harlay, J. [25]

Harris, P.P. [57,51]

Hart, J.L. [93]

Hartley, A.E. [16]

Hashimoto, H. [72]

Hasselmann, K. [49]

Hattenschwiler, S. [55]

Haugen-Korzyra, K.L. [125]

Hausman, J.A. [97]

Heemann, C. [25]

Heimann, M. [45,67,88]

Hennessy, K. [14]

Heyder, U. [6]

Hicke, J.A. [71] 
Hickler, T. [56,78]

Hiederer, R. [118,119]

Hiley, J.C. [125]

Hirst, A.C. $[30,18]$

Hoffmann, L. [25]

Hogg, E.H. [58]

Holdren, J.P. [98]

Holland, E. [71]

Hooss, G. [49]

Houghton, R.A. [45]

House, J.I. [45]

Hulme, M. [63]

Huntingford, C. [57,51]

Hutyra, L. [79]

Ikaga, T. [105]

Isbell, R.F. [114]

Ishida, A. [19]

Ishimatsu, A. [26]

Izaurralde, R.C. [125]

Jackson, R.B. [129]

Jacquet, S. [25]

Jans, D.C. [125]

Janssens, I.A. [81]

Jaramillo, V.J. [88]

Jenkins, J.C. [71]

Jenkinson, D.S. [120,130,131]

John, J. [50]

Johns, T.C. [64]
Jolly, W.M. [72]

Jones, C. [50]

Jones, C.D. $[57,42,51]$

Jones, P.D. [63]

Jones, R. [119]

Jones, R.J.A. [118]

Joos, F. [50,61,49,19]

Kainuma, M. [106]

Kankaanpaa, S. [119]

Kaplan, J.O. [70]

Karl, T.R. [40]

Kasibhatla, P. [77,66]

Kasischke, E. [66]

Kasischke, E.S. [77]

Kato, S. [105]

Kato, T. [50]

Katterer, T. [122]

Kawamiya, M. [50]

Keel, S.G. [55]

Keeling, C.D. $[67,72]$

Keeling, R.F. [35,67]

Keller, M. [79]

Kern, J.S. [115]

Key, R.M. [34,36,19]

Kheshgi, H. [67]

Kheshgi, H.S. [88]

Kikkawa, T. [26]

Kindermann, G. [15] 
King, A.W. [124,110]

Kirchhoff, V. [79]

Kita, J. [26]

Klooster, S. [73]

Knorr, W. [50]

Koch, G.W. [54]

Kolp, P. [106]

Korner, C. [55]

Kraxner, F. [135]

Kubiske, M.E. [54]

Kucharik, C. [43]

Kuhnz, L. [24]

Kumar, V. [73]

Kurz, W.A. [17]

Ladd, J.N. [123,109,132]

Langenbuch, M. [29]

Le Quere, C. $[67,36,88]$

Lebel, L. [99]

Leemans, R. [60]

Levis, S. [70]

Lieth, H. [126]

Lindner, M. [119]

Lindsay, A.M. [111]

Lindsay, K. [50,19]

Lloyd, J. [84]

Lomas, M.R. $[43,48]$

London, B. [100]

Los, S. [71]
Lovera, C.F. [24]

Lucht, W. $[80,7,52,56,76,6,47,70,87]$

Luo, Y.Q. [53]

Maier-Reimer, E. [36,19]

Marchetti, C. [32]

Marion, G.M. [16]

Masui, T. [41]

Matear, R. [2,19]

Matear, R.J. [18,34]

Matross, D.M. [79]

Matsumoto, K. [4]

Matthews, H.D. [50]

McCallum, I. [15,135]

McGill, W.B. [125]

McKinley, G. [65]

McNeil, B.I. [34,2,5]

McPhaden, J. [69]

McSweeney, K. [136]

Menton, M. [79]

Meyer, J. [119]

Meyer, R. [49]

Michalsky, J.J. [75]

Mikolajewicz, U. [36]

Miller, J.B. [77]

Miller, S.D. [79]

Millero, F.J. [39]

Milligan, C.L. [21]

Mitchell, J.F.B. [64] 
Mitchell, M.J. [16]

Monfray, P. [46,36,19]

Montanarella, L. [118,119]

Mooney, H.A. [129]

Moorcroft, P.R. [62]

Mouchet, A. [19]

Munger, J.W. [75,79]

Murakami, S. [105]

Myneni, R.B. [80,76,72,73]

Najjar, R.G. [19]

Nakicenovic, N. [106]

Naumburg, E.S. [54]

Neilson, R.P. [59]

Nejstgaard, J. [25]

Nemani, R.R. [72]

New, M.G. [63]

Nix, H.A. [113]

Norby, R.J. [53,16]

Norman, J. [136]

Obersteiner, M. [134,15,135]

Ofarrell, S.P. [30,31]

Oikawa, T. [133]

Olsen, S. [66]

Orr, J.C. $[36,19]$

Pacala, S. [33]

Palmer, J. [36]

Parton, W.J. [121]

Pathe, C. [87]
Pedersen, M.F. [27,28]

Pelez-Riedl, S. [55]

Peltzer, E.T. [24]

Peng, T.H. [110]

Pepin, S. [55]

Peylin, P. [67,65]

Piper, S.C. $[67,72]$

Pittock, A.B. [113]

Pizay, M.D. [25]

Plattner, G.K. $[49,19]$

Polglase, P.J. [127]

Pomaz, V.L. [102]

Portner, H.O. [29]

Post, W.M. $[14,124,110]$

Potter, C.S. $[80,73]$

Prentice, I.C. $[43,61,45,49,67,76,88,47,70]$

Probert, M.E. [114]

Pyle, E.H. [79]

Quere, C.L. [65]

Raddatz, T. [50]

Ramankutty, N. [43,45,136]

Rametsteiner, E. [15]

Randerson, J. [66]

Randerson, J.T. [71,77]

Rasmussen, P.E. [121]

Rayner, P. [44,50,65]

Rayner, P.J. [67]

Reginster, I. [118,119] 
Reich, P.B. [54]

Reick, C. [50]

Reipschlager, A. [29]

Reynolds, R. [68]

Riahi, K. [106,135]

Ribas-Carbo, M. [83]

Rice, A.H. [79]

Riebesell, U. [25]

Ringler, D.C. [41]

Robertson, G.P. [109,108]

Rochelle-Newall, E. [25]

Rodgers, K.B. [19]

Roeckner, E. [50]

Rokityanskiy, D. [135]

Rosa, E. [95,104]

Rounsevell, M. [119]

Rounsevell, M.D.A. [118]

Rudolf, B. [87]

Ruedy, R. [68]

Running, S.R. [72]

Rustad, L.E. [16]

Sabine, C.L. [36,19]

Safir, G.R. [108]

Salameh, P.K. [37]

Saleska, S.R. [79]

Sarmiento, J.L. $[34,36,19]$

Sato, M. [68]

Schaphoff, S. [52,56,6,47]
Schellnhuber, H.J. [102,8]

Schienke, E. [13]

Schlesinger, W.H. [107]

Schlitzer, R. [19]

Schneider, U. [25]

Schnur, R. [50]

Scholes, R.J. [88]

Scholz, S. [11]

Schulze, E.D. [129]

Schulze, K. [41]

Schwarz, A.G. [58]

Scipal, K. [87]

Seibel, B.A. [22,23]

Senior, C.A. [64]

Shandra, J.M. [100]

Shiraishi, Y. [105]

Siegwolf, R.T.W. [55]

Silva, H. [79]

Sitch, S. $[43,52,49,76,65,47,70]$

Slater, R.D. [19]

Smith, B. $[43,78,76,70]$

Smith, J.U. [118,119]

Smith, P. [118,119]

Smith, S.D. [54]

Snitzler, K.G. [50]

Socolow, R. [33]

Sohlberg, R. [86]

Sonnerup, R.E. [38] 
Soule, P. [93]

Spain, A.V. [114]

Spall, S.A. [42]

Steffen, W. [3]

Still, C.J. [77]

Strassmann, K. [50]

Strengers, Y. [101]

Sugita, S. [78]

Svirejeva-Hopkins, A. [102,8]

Svirezhev Yu, M. [90]

Sykes, M.T. [78,70]

Tan, P. [73]

Taylor, J.A. [84]

Taylor, N.K. [36]

Terbrueggen, A. [25]

Thonicke, K. [70]

Toggweiler, J.R. [36]

Totterdell, I.J. [42,19]

Townshend, J.R.G. [86]

Trenbreth, K.E. [40]

Tucker, C. [71]

Tucker, C.J. [80,72]

Umemiya, C. [9]

Urbanski, S.P. [75]

Van der Werf, G. [66]

Van der Werf, G.R. [77]

van Veen, J.A. [132]

van Vuuren, D. [41]
Venevsky, S. [70]

von Bloh, W. [50]

von Caemmerer, S. [82]

Wagner, W. [56,87]

Waley, P. [103]

Walker, K. [78]

Walker, S.J. [37]

Walker, T.W. [112]

Wallace, D.W.R. [88]

Walsh, P.J. [22,23,21]

Walz, P. [24]

Wang, Y.P. [127]

Wattenbach, M. [118,119]

Watterson, I.G. [31]

Weaver, A.J. [50]

Weirig, M.F. [19]

Weiss, R.F. [37]

Whaling, P.J. [24]

White, A. [43]

White, J.W.C. [77]

Whooley, O. [100]

Wickett, M.E. [20]

Wild, A. [131]

Williamson, J. [100]

Wofsy, S.C. $[75,79]$

Wood, R.A. [64]

Woodward, F.I. $[43,48]$

Wullschleger, S.D. [124] 
Yamagata, Y. [133,135]

Yamanaka, Y. [19]

Yool, A. [19]

York, R. [104]

Yoshida, Y. [12]

Yoshikawa, C. [50]

Young-Molling, C. [43]

Zaehle, S. [118,119]

Zeng, N. [50]

Zondervan, I. [25]

Zotz, G. [55]

\section{Additional material}

\section{Additional File 1}

References to the journal articles cited in the first volume of the journal. This file is formatted for importing references into a reference database. Click here for file

[http://www.biomedcentral.com/content/supplementary/17500680-2-1-S1.xml]

\section{Additional File 2}

The list of words and wordings selected from the articles published in the first volume of the journal.

Click here for file

[http://www.biomedcentral.com/content/supplementary/17500680-2-1-S2.txt]

\section{References}

I. Dickinson RE: Welcome to Carbon Balance and Management. Carbon Balance and Management 2006, I:I.

2. McNeil BI, Matear R: Projected climate change impact on oceanic acidification. Carbon Balance and Management 2006, I:2.

3. Steffen W: The Anthropocene, global change and sleeping giants: where on Earth are we going? Carbon Balance and Management 2006, I:3.

4. Matsumoto K: A psychological effect of having a potentially viable sequestration strategy. Carbon Balance and Management 2006, I:4.

5. McNeil BI: Significance of the oceanic $\mathrm{CO}_{2}$ sink for national carbon accounts. Carbon Balance and Management 2006, I:5.

6. Lucht W, Schaphoff S, Erbrecht T, Heyder U, Cramer W: Terrestrial vegetation redistribution and carbon balance under climate change. Carbon Balance and Management 2006, I:6.

7. Erbrecht $T$, Lucht $W$ : Impacts of large-scale climatic disturbances on the terrestrial carbon cycle. Carbon Balance and Management 2006, I:7

8. Svirejeva-Hopkins A, Schellnhuber $\mathrm{HJ}$ : Modelling carbon dynamics from urban land conversion: fundamental model of city in relation to a local carbon cycle. Carbon Balance and Management 2006, I:8.

9. Umemiya C: Improving GHG inventories by regional information exchange: a report from Asia. Carbon Balance and Management 2006, I:9.

10. Alexandrov GA: The purpose of peer review in the case of an open-access publication. Carbon Balance and Management 2006. I:I0.

II. Scholz S: The POETICs of industrial carbon dioxide emissions in Japan: an urban and institutional extension of the IPAT identity. Carbon Balance and Management 2006, I: I I

12. Yoshida Y: Development of air conditioning technologies to reduce $\mathrm{CO}_{2}$ emissions in the commercial sector. Carbon Balance and Management 2006, I:I2.

13. Canan P, Schienke E: Responsibility, opportunity, and vision for higher education in urban and regional carbon management. Carbon Balance and Management 2006, I:I3.

14. Grace PR, Post WM, Hennessy K: The potential impact of climate change on Australia's soil organic carbon resources. Carbon Balance and Management 2006, I:|4.

15. Kindermann G, Obersteiner M, Rametsteiner E, McCallum I: Predicting the deforestation-trend under different carbonprices. Carbon Balance and Management 2006, I:I5.

16. Rustad LE, Campbell J, Marion GM, Norby RJ, Mitchell MJ, Hartley AE, Cornelissen JHC, Gurevitch J, GCTE NEWS: A meta-analyses of the response of soil respiration, net $\mathbf{N}$ mineralization, and aboveground plant growth to experimental ecosystem warming. Oecologia 2000, 126:543-562.

17. Kurz WA, Apps MJ: A 70-year retrospective analysis of carbon fluxes in the Canadian forest sector. Ecological Applications 1999 , 9:526-547.

18. Matear RJ, Hirst AC: Climate change feedback on the future oceanic $\mathrm{CO}_{2}$ uptake. Tellus Ser B-Chem Phys Meteorol 1999. I I:722-733

19. Orr JC, Fabry VJ, Aumont O, Bopp L, Doney SC, Feely RA, Gnanadesikan A, Gruber N, Ishida A, Joos F, Key RM, Lindsay K, MaierReimer E, Matear R, Monfray P, Mouchet A, Najiar RG, Plattner GK, Rodgers KB, Sabine CL, Sarmiento JL, Schlitzer R, Slater RD, Totterdell I], Weirig MF, Yamanaka Y, Yool A: Anthropogenic ocean acidification over the twenty-first century and its impact on calcifying organisms. Nature 2005, 437:68I-686.

20. Caldeira K, Wickett ME: Anthropogenic carbon and ocean pH. Nature 2003, 425:365.

21. Walsh PJ, Milligan CL: Coordination of Metabolism and Intracellular Acid-Base Status - Ionic Regulation and Metabolic Consequences. Canadian Journal of Zoology-Revue Canadienne De Zoologie 1989, 67:2994-3004.

22. Seibel BA, Walsh PJ: Carbon cycle - Potential, impacts of CO injection on deep-sea biota. Science 2001, 294:319-320.

23. Seibel BA, Walsh PJ: Biological impacts of deep-sea carbon dioxide injection inferred from indices of physiological performance. Journal of Experimental Biology 2003, 206:64I-650.

24. Barry JP, Buck KR, Lovera CF, Kuhnz L, Whaling PJ, Peltzer ET, Walz $P$, Brewer PG: Effects of direct ocean $\mathrm{CO}_{2}$ injection on deepsea meiofauna. Journal of Oceanography 2004, 60:759-766.

25. Engel A, Zondervan I, Aerts K, Beaufort L, Benthien A, Chou L, Delille B, Gattuso JP, Harlay J, Heemann C, Hoffmann L, Jacquet S, Nejstgaard J, Pizay MD, Rochelle-Newall E, Schneider U, Terbrueggen A, Riebesell $\mathrm{U}$ : Testing the direct effect of $\mathrm{CO}_{2}$ concentration on a bloom of the coccolithophorid Emiliania huxleyi in mesocosm experiments. Limnology and Oceanography 2005, 50:493-507.

26. Kikkawa $\mathrm{T}$, Ishimatsu $\mathrm{A}$, Kita J: Acute $\mathbf{C O}_{2}$ tolerance during the early developmental stages of four marine teleosts. Environmental Toxicology 2003, I 8:375-382.

27. Pedersen MF, Hansen PJ: Effects of high pH on a natural marine planktonic community. Marine Ecology-Progress Series 2003 , 260: $|9-3|$.

28. Pedersen MF, Hansen PJ: Effects of high pH on the growth and survival of six marine heterotrophic protists. Marine EcologyProgress Series 2003, 260:33-4I.

29. Portner HO, Langenbuch M, Reipschlager A: Biological impact of elevated ocean $\mathrm{CO}_{2}$ concentrations: Lessons from animal physiology and earth history. Journal of Oceanography 2004, 60:705-718 
30. Hirst AC, Gordon HB, Ofarrell SP: Global warming in a coupled climate model including oceanic eddy-induced advection. Geophysical Research Letters 1996, 23:336I-3364.

31. Watterson IG, Ofarrell SP, Dix MR: Energy and water transport in climates simulated by a general circulation model that includes dynamic sea ice. Journal of Geophysical Research-Atmospheres 1997, I 02(D I 0): I I027-I I 037.

32. Marchetti $\mathrm{C}$ : On geoengineering and the $\mathbf{C O}_{2}$ problem. Climatic Change 1977, I:59-68.

33. Pacala S, Socolow R: Stabilization wedges: Solving the climate problem for the next $\mathbf{5 0}$ years with current technologies. Science 2004, 305:968-972.

34. McNeil BI, Matear RJ, Key RM, Bullister JL, Sarmiento JL: Anthropogenic $\mathrm{CO}_{2}$ uptake by the ocean based on the global chlorofluorocarbon data set. Science 2003, 299:235-239.

35. Keeling RF, Garcia HE: The change in oceanic $\mathbf{O}_{2}$ inventory associated with recent global warming. Proc Natl Acad Sci USA 2002, 99:7848-7853.

36. Orr JC, Maier-Reimer E, Mikolajewicz U, Monfray P, Sarmiento JL, Toggweiler JR, Taylor NK, Palmer J, Gruber N, Sabine CL, Le Quere C, Key RM, Boutin J: Estimates of anthropogenic carbon uptake from four three-dimensional global ocean models. Glob Biogeochem Cycle 2001, 15:43-60.

37. Walker SJ, Weiss RF, Salameh PK: Reconstructed histories of the annual mean atmospheric mole fractions for the halocarbons CFC-II, CFC-I2, CFC-II3 and carbon tetrachloride. Journal of Geophysical Research 2000, I 05:14, 285-I4, 296.

38. Sonnerup RE: On the relations among CFC derived water mass ages. Geophys Res Lett 200I, 28:1739-1742.

39. Dickson AG, Millero FJ: A Comparison of the Equilibrium-Constants for the Dissociation of Carbonic-Acid in Seawater Media. Deep-Sea Research Part a-Oceanographic Research Papers 1987, 34: I733-I743.

40. Karl TR, Trenbreth KE: Modern global climate change. Science 2003, 302: $1719-1723$

4I. Alcamo J, van Vuuren D, Ringler DC, Cramer W, Masui T, Alder J, Schulze K: Changes in nature's balance sheet: model-based estimates of future worldwide ecosystem services. Ecology and Society 2005, 10:19.

42. Cox PM, Betts RA, Jones CD, Spall SA, Totterdell IJ: Acceleration of global warming due to carbon-cycle feedbacks in a coupled climate model. Nature 2000, 408: |84-|87.

43. Cramer W, Bondeau A, Woodward FI, Prentice IC, Betts RA, Brovkin V, Cox PM, Fisher V, Foley JA, Friend AD, Kucharik C, Lomas MR, Ramankutty N, Sitch S, Smith B, White A, Young-Molling C: Global response of terrestrial ecosystem structure and function to $\mathrm{CO}_{2}$ and climate change: Results from six dynamic global vegetation models. Global Change Biology 2001:357-373.

44. Friedlingstein P, Dufresne JL, Cox PM, Rayner P: How positive is the feedback between climate change and the carbon cycle? Tellus 2003, 55B:692-700.

45. House JI, Prentice IC, Ramankutty N, Houghton RA, Heimann M: Reconciling apparent inconsistencies in estimates of terrestrial $\mathbf{C O}_{2}$ sources and sinks. Tellus 2003, 55B:345-363.

46. Berthelot M, Friedlingstein P, Ciais P, Dufresne JL, Monfray P: How uncertainties in future climate change predictions translate into future terrestrial carbon fluxes. Glob Change Biol 2005 , I:959-970.

47. Schaphoff S, Lucht W, Gerten D, Sitch S, Cramer W, Prentice IC: Terrestrial biosphere carbon storage under alternative climate projections. Climatic Change 2006.

48. Woodward FI, Lomas MR: Vegetation dynamics - simulating responses to climatic change. Biol Rev 2004, 79:643-670.

49. Joos F, Prentice IC, Sitch S, Meyer R, Hooss G, Plattner GK, Gerber $\mathrm{S}$, Hasselmann K: Global warming feedbacks on terrestrial carbon uptake under the Intergovernmental Panel on Climate Change (IPCC) emission scenarios. Global Biogeochemical Cycles 2001, I 5:891-907.

50. Friedlingstein P, Cox P, Betts R, Bopp L, von Bloh W, Brovkin V, Cadule P, Doney S, Eby M, Fung I, Bala G, John J, Jones C, Joos F, Kato T, Kawamiya M, Knorr W, Lindsay K, Matthews HD, Raddatz T, Rayner P, Reick C, Roeckner E, Snitzler KG, Schnur R, Strassmann K, Weaver AJ, Yoshikawa C, Zeng N: Climate-carbon cycle feedback analysis: Results from the C4MIP model intercomparison. J Clim 2006, 19:3337-3353.
5I. Cox PM, Betts RA, Collins M, Harris PP, Huntingford C, Jones CD: Amazonian forest dieback under climate-carbon cycle projections for the 2 I st century. Theoretical and Applied Climatology 2004, 78:137-I56.

52. Gerten D, Schaphoff S, Haberlandt U, Lucht W, Sitch S: Terrestrial vegetation and water balance: Hydrological evaluation of a dynamic global vegetation model. Journal of Hydrology 2004, 286:249-270.

53. Norby RJ, Luo YQ: Evaluating ecosystem responses to rising atmospheric $\mathrm{CO}_{2}$ and global warming in a multi-factor world. New Phytologist 2004, I 62:28I-293.

54. Ellsworth DS, Reich PB, Naumburg ES, Koch GW, Kubiske ME, Smith SD: Photosynthesis, carboxylation and leaf nitrogen responses of 16 species to elevated $\mathrm{pCO}_{2}$ across four free-air $\mathrm{CO}_{2}$ enrichment experiments in forest, grassland and desert. Global Change Biology 2004, 1 0:21 21 -21 38.

55. Korner C, Asshoff R, Bignucolo O, Hattenschwiler S, Keel SG, PelezRiedl S, Pepin S, Siegwolf RTW, Zotz G: Carbon flux and growth in mature deciduous forest trees exposed to elevated $\mathrm{CO}_{2}$. Science 2005, 309:1360-1362.

56. Gerten D, Lucht W, Schaphoff S, Cramer W, Hickler T, Wagner W: Hydrologic resilience of the terrestrial biosphere. Geophys Res Lett 2005, 32:

57. Betts RA, Cox PM, Harris PP, Huntingford C, Jones CD: The role of ecosystem-atmosphere interactions in simulated Amazon forest dieback under global climate warming. Theoretical and Applied Climatology 2004, 78: I57-I75.

58. Hogg EH, Schwarz AG: Regeneration of planted conifers across climatic moisture gradients on the Canadian prairies: implications for distribution and climate change. Journal of Biogeography 1997, 24:527-534.

59. Neilson RP, Drapek RJ: Potentially complex biosphere responses to transient global warming. Global Change Biology 1998, 4:505-52|.

60. Leemans R, Eichhout B: Another reason for concern: regional and global impacts on ecosystems for different levels of climate change. Global Environmental Change 2004, I 4:2 I9-228.

61. Gerber S, Joos F, Prentice IC: Sensitivity of a dynamic global vegetation model to climate and atmospheric $\mathrm{CO}_{2}$. Global Change Biology 2004, 1 0: 1223-1239.

62. Moorcroft PR: How close are we to a predictive science of the biosphere? Trends in Ecology and Evolution 2006, 21 :400-407.

63. New MG, Hulme M, Jones PD: Representing twentieth-century space-time climate variability, Part II, Development of I90 I1996 monthly grids of terrestrial surface climate. Journal of Climate 2000, 13:2217-2238.

64. Gordon C, Cooper C, Senior CA, Banks HT, Gregory JM, Johns TC, Mitchell JFB, Wood RA: The simulation of SST, sea ice extents and ocean heat transports in a version of the Hadley Centre coupled model without flux adjustments. Climate Dynamics 2000, 16:147-168.

65. Peylin P, Bousquet P, Quere CL, Sitch S, Friedlingstein P, McKinley G, Gruber N, Rayner $P$, Ciais $P$ : Multiple constraints on regional $\mathrm{CO}_{2}$ flux variations over land and oceans. Global Biogeochemical Cycles 2005:19.

66. Van der Werf G, Randerson J, Collatz G, Giglio L, Kasibhatla P, Arellano A, Olsen S, Kasischke E: Continental-Scale Partitioning of Fire Emissions During the 1997 to 200 I El Nino/La Nina Period. Science 2004, 303:73-76.

67. Le Quere C, Aumont OL, Bopp L, Bousquet P, Ciais P, Francey R, Heimann M, Keeling CD, Keeling RF, Kheshgi H, Peylin P, Piper SC, Prentice IC, Rayner PJ: Two decades of ocean $\mathrm{CO}_{2}$ sink and variability. Tellus 2003, 55B:649-656.

68. Hansen J, Ruedy R, Sato M, Reynolds R: Global surface air temperature in 1995: Return to pre-Pinatubo level. Geophysical Research Letters 1996, 23:1665-1668.

69. McPhaden J: Genesis and Evolution of the I997-98 EI Nino. Science 1999, 283:950-954.

70. Sitch S, Smith B, Prentice IC, Arneth A, Bondeau A, Cramer W, Kaplan JO, Levis S, Lucht W, Sykes MT, Thonicke K, Venevsky S: Evaluation of ecosystem dynamics, plant geography and terrestial carbon cycling in the LPJ dynamic global vegetation model. Global Change Biology 2003, 9:16I-185.

7I. Hicke JA, Asner GP, Randerson JT, Tucker C, Los S, Birdsey R, Jenkins JC, Field C, Holland E: Satellite-derived increases in net primary 
productivity across North America, 1982-1998. Geophysical Research Letters 2002, 29:69-73.

72. Nemani RR, Keeling CD, Hashimoto H, Jolly WM, Piper SC, Tucker C], Myneni RB, Running SR: Climate-Driven Increases in Global Terrestial Net Primary Production from 1982 to 1999. Science 2003, 300:1560-1563.

73. Potter CS, Klooster S, Myneni RB, Genovese V, Tan P, Kumar V: Continental-scale comparisons of terrestial carbon sinks estimated from satellite data and ecosystem modeling 1982 1998. Global and Planetary Change 2003, 39:20I-2I3.

74. Angert A, Biraud S, Bonfils C, Buermann W, Fung I: $\mathrm{CO}_{2}$ seasonality indicates origins of post-Pinatubo sink. Geophysical Research Letters 2004:3I.

75. Gu L, Baldocchi DD, Wofsy SC, Munger JW, Michalsky JJ, Urbanski SP, Boden TA: Response of a Deciduous Forest to the Mount Pinatubo Eruption: Enhanced Photosynthesis. Science 2003, 299:2035-2038.

76. Lucht W, Prentice IC, Myneni RB, Sitch S, Friedlingstein P, Cramer W, Bousquet P, Buermann W, Smith B: Climate Control of the HighLatitude Vegetation Greening Trend and Pinatubo Effect. Science 2002, 296: 1687-I689.

77. Randerson JT, Van der Werf GR, Collatz GJ, Giglio L, Still CJ, Kasibhatla P, Miller JB, White JWC, DeFries RS, Kasischke ES: Fire emissions from $\mathrm{C} 3$ and $\mathrm{C} 4$ vegetation and their influence on interannual variability of atmospheric $\mathrm{CO}_{2}$ and delta $13 \mathrm{CO}_{2}$. Global Biogeochemical Cycles 2005:19.

78. Hickler T, Smith B, Sykes MT, Davis M, Sugita S, Walker K: Using a generalized vegetation model to simulate vegetation dynamics in northeastern USA. Ecology 2004, 85:519-530.

79. Saleska SR, Miller SD, Matross DM, Goulden ML, Wofsy SC, da Rocha HR, de Camargo PB, Crill P, Daube BC, de Freitas HC, Hutyra L, Keller M, Kirchhoff V, Menton M, Munger JW, Pyle EH, Rice AH, Silva H: Carbon in Amazon Forests: Unexpected Seasonal Fluxes and Disturbance-Induced Losses. Science 2003, 302: I554-I557.

80. Buermann W, Anderson B, Tucker CJ, Dickinson RE, Lucht W, Potter CS, Myneni RB: Interannual covariability in Northern Hemisphere air temperetures and greenness associated with $\mathrm{El}$ Nino-Southern Oscillation and the Arctic Oscillation. Journal of Geophysical Research 2003, 108:4396-444I.

8I. Davidson EA, Janssens IA: Temperature sensitivity of soil carbon decomposition and feedbacks to climate change. Nature 2006, 440:165-173

82. Farquhar GD, von Caemmerer S, Berry JA: A biochemical model of photosynthetic $\mathrm{CO}_{2}$ assimilation in leaves of $\mathrm{C} 3$ species. Planta 1980, 149:78-90.

83. Collatz GJ, Ribas-Carbo M, Berry JA: Coupled photosynthesisstomatal conductancemodel for leaves of C4 plants. Australian Journal of Plant Physiology 1992, 19:5 19-538.

84. Lloyd J, Taylor JA: On the temperature dependence of soil respiration. Functional Ecology 1994, 8:3 I5-323.

85. Foley JA: An equilibrium model of the terrestrial carbon budget. Tellus 2005, 47B:310-319.

86. Hansen M, DeFries R, Townshend JRG, Sohlberg R: Global land cover classification at $\mathbf{I ~} \mathbf{~ k m}$ spatial resolution using a classification tree approach. International Journal of Remote Sensing 2000 , 2I:|33|-|364.

87. Wagner W, Scipal K, Pathe C, Gerten D, Lucht W, Rudolf B: Evaluation of the agreement between the first global remotely sensed soil moisture data with model and precipitation data. Journal of Geophysical Research 2003, I08: I-10.

88. Prentice IC, Farquhar GD, Fasham MJR, Goulden ML, Heimann M, Jaramillo VJ, Kheshgi HS, Le Quere C, Scholes RJ, Wallace DWR: The carbon cycle and atmospheric carbon dioxide. Climate Change 200 I: The Scientific Basis Contribution of Working Group I to the Third Assessment Report of the Intergovernmental Panel on Climate Change 200I, I:I85-225

89. Bazilevich NI: Biogeochemisty of the Earth and functional models of exchange processes in natural ecosystems. Modern Concepts and Problem of Biogeochemistry (Proceedings of the Biogeochemical Laboratory), Nauka Moscow 1979, 17:56-73.

90. Svirezhev Yu M: Simple spatially distributed model of the global carbon cycle and its dynamic properties. Ecol Modelling 2002, 155:53-69.

91. Catton WR, Dunlap RE: Environmental Sociology: A New Paradigm. American Sociologist 1978, 13:41-49.
92. Cramer JC: Population Growth and Air Quality in California. Demography 1998, 35:45-56.

93. Hart JL, Soule P: Does I = PAT Work in Local Places? Professiona Geographer 2000, 52:1-10.

94. Diamond L: Rethinking Civil Society. Journal of Democracy 1994, 5:5-17.

95. Dietz T, Rosa E: Rethinking the Environmental Impacts of Population, Affluence and Technology. Human Ecology Review 1994, I.2:277-300.

96. Duncan OD: From Social System to Ecosystem. Sociological Inquiry 196I, 31: I 40-149.

97. Hausman JA: Specification Tests in Econometrics. Econometrica 1978, 46:|25I-I27|.

98. Holdren JP, Ehrlich P: Human Population and the Global Environment. American Scientist 1974, 62:282-292.

99. Lebel L: Social Change and $\mathrm{CO}_{2}$ Stabilization: Moving Away from Carbon Cultures. SCOPE: The Global Carbon Cycle 2004, 62:37I-383.

100. Shandra JM, London B, Whooley O, Williamson J: International Nongovernmental Organizations and CarbonDioxide Emissions in the Developing World: A Quantitative, CrossNational Analysis. Sociological Inquiry 2004, 74:520-545.

10I. Strengers Y: Environmental Culture Change in LocalGovernment: A Practised Perspective from the International Council for Local Environmental Initiatives - Australia/New Zealand. Local Environment 2004, 9:62I-628.

102. Svirejeva-Hopkins A, Schellnhuber HJ, Pomaz VL: Urbanised Territories as a Specific Component of the Global Carbon Cycle. Ecological Modelling 2004, I 73:295-3 I2.

103. Waley P: Ruining and Restoring Rivers: The State and Civil Society in Japan. Pacific Affairs 2005, 78:.

104. York R, Rosa E, Dietz T: STIRPAT, IPAT and ImPACT: Analytic Tools for Unpacking the Driving Force of Environmental Impacts. Ecological Economics 2003, 46:35I-365.

105. Ikaga T, Murakami S, Kato S, Shiraishi Y: Estimation of $\mathrm{CO}_{2}$ Emission Associated with Building Construction and Operation till 2050 in Japan: Study on Social Life Cycle Assessment of Buildings and Cities. J Archt Plann Environ Eng 2000, 535:53-58.

106. Nakicenovic N, Kolp P, Riahi K, Kainuma M, Hanaoka T: Assessment of Emissions Scenarios Revisited. Environmental Economics and Policy Studies 2006, 7:137-173.

107. Schlesinger WH: Carbon sequestration in soils. Science 1999 284:2095

108. Grace PR, Colunga-Garcia M, Gage SH, Robertson GP, Safir GR: The potential impact of agricultural management and climate change on soil organic carbon resources in terrestrial ecosystems of the North Central Region of the United States. Ecosystems 2006, 9:816-827.

109. Grace PR, Ladd JN, Robertson GP, Gage SH: SOCRATES - a simple model for predicting long-term changes in soil organic carbon in terrestrial ecosystems. Soil Biol Biochem 2005, 38: $1172-1176$.

1 10. Post WM, Peng TH, Emanuel WR, King AW, Dale VH, DeAngelis DL: The global carbon cycle. Amer Scient 1990, 78:310-326.

III. Lindsay AM: Are Australian soils different? Proc Ecol Soc Aust 1985, 14:83-97.

112. Walker TW, Adams AFR: Studies on soil organic matter: I. Influence of phosphorus content of parent material on accumulation of carbon, nitrogen, sulphur and organic phosphorus in grassland soils. Soil Sci 1958, 85:307-318.

I 13. Pittock $A B, N i x$ HA: The effect of changing climate on Australian biomass production - a preliminary study. Clim Change 1986, 8:243-255.

I 4. Spain AV, Isbell RF, Probert ME: Soil organic matter. Soils: An Australian Viewpoint 1983:55 I-563.

115. Kern JS: Spatial patterns of soil organic carbon in the contiguous United States. Soil Sci Soc Am J 1994, 58:439-455.

II6. Barrett DJ: Steady state net primary productivity, carbon stocks and mean residence time of carbon in the Australian terrestrial biosphere. Global Biogeochem Cycles 2002, I I 6: I I 08

117. Gifford RM: Implications of the globally increasing atmospheric $\mathrm{CO}_{2}$ concentration and temperature for the Australian terrestrial carbon budget: integration using a simple model. Aust J Bot 1992, 40:527-543.

118. Smith JU, Smith P, Wattenbach M, Zaehle S, Hiederer R, Jones RJA, Montanarella L, Rounsevell MDA, Reginster I, Ewert F: Projected 
changes in mineral soil carbon of European croplands and grasslands, 1990-2080. Global Change Biol 2005, I I:2 I4I-2I 52 .

119. Smith P, Smith JU, Wattenbach M, Meyer J, Lindner M, Zaehle S, Hiederer R, Jones R, Montanarella L, Rounsevell M, Reginster I, Kankaanpaa S: Projected changes in mineral soil carbon of European forests, 1990-2100. Canadian J Soil Sci 2006, 86:159-169.

120. Coleman K, Jenkinson DS: RothC 26.3-A model for the turnover of carbon in soil. Evaluation of Soil Organic Matter Models Using LongTerm Datasets (NATO ASI Series I Vol 38) 1990:237-246.

121. Parton W], Rasmussen PE: Long term effects in crop management-fallow: II. Century model formulation. Soil Sci Soc Am J 1994, 58:530-536.

122. Katterer T, Andren O: The ICBM family of analytically solved models of soil carbon, nitrogen and microbial biomass dynamics - descriptions and application examples. Ecol Mod 200I, 136:191-207.

123. Amato M, Ladd JN: Decomposition of I4C-labelled glucose and legume materials in soils: Properties influencing the accumulation of organic residue $\mathbf{C}$ and microbial biomass $\mathbf{C}$. Soil Biol Biochem 1992, 27:455-464.

124. King AW, Post WM, Wullschleger SD: The potential response of terrestrial carbon storage to changes in climate and atmospheric $\mathrm{CO}_{2}$. Climatic Change 1997, 35:199-227.

125. Izaurralde RC, Haugen-Korzyra KL, Jans DC, McGill WB, Grant RF, Hiley JC: Soil C dynamics: Measurement, simulation and siteto-region scale-up. Assessment Methods of Soil Carbon 200I:553-575.

126. Lieth H: Modeling the primary productivity of the world. Primary Productivity of the Biosphere 1975:237-263.

127. Polglase PJ, Wang YP: Potential $\mathbf{C O}_{2}$-enhanced carbon storage by the terrestrial biosphere. Aust J Bot 1992, 40:64I-656.

128. Cure JD, Acock B: Crop responses to carbon dioxide doubling: a literature survey. Agric For Meteor 1986, 38: I 27-I 45.

129. Jackson RB, Canadell J, Erlinger JR, Mooney HA, Dala OE, Schulze ED: A global analysis of root distributions for terrestrial biomes. Oecolog 1996, 108:389-4II.

130. Jenkinson DS: The turnover of organic carbon and nitrogen in soil. Phil Trans Roy Soc London [B] 1990, 329:36 I-368.

131. Jenkinson DS, Adams DE, Wild A: Model estimates of $\mathrm{CO}_{2}$ emissions from soil in response to global warming. Nature 1991, 35 I:304-306.

132. Ladd JN, Amato M, Grace PR, van Veen JA: Simulation of I4C turnover through the microbial biomass in soils incubated with I4C-labelled plant residues. Soil Biol Biochem 1995, 27:777-783.

133. Alexandrov GA, Yamagata Y, Oikawa T: Towards a model for projecting Net Ecosystem Production of the world forests. Ecological Modelling 1999, I 23: 183-191.

134. Benitez PC, Obersteiner M: Site identification for carbon sequestration in Latin America: A grid-based economic approach. Forest Policy and Economics 2006, 8:636-65I.

135. Obersteiner M, Alexandrov G, Benitez PC, McCallum I, Kraxner F, Riahi K, Rokityanskiy D, Yamagata Y: Global Supply of Biomass for Energy and Carbon Sequestration from Afforestation/ Reforestation Activities. Mitigation and Adaptation Strategies for Global Change 2006: I38I-2386.

136. Ramankutty N, Foley JA, Norman J, McSweeney K: The global distribution of cultivable lands: current patterns and sensitivity to possible climate change. Global Ecology \& Biogeography 2002, I I:377-392.
Publish with Biomed Central and every scientist can read your work free of charge

"BioMed Central will be the most significant development for disseminating the results of biomedical research in our lifetime. "

Sir Paul Nurse, Cancer Research UK

Your research papers will be:

- available free of charge to the entire biomedical community

- peer reviewed and published immediately upon acceptance

- cited in PubMed and archived on PubMed Central

- yours - you keep the copyright

Submit your manuscript here:

http://www.biomedcentral.com/info/publishing_adv.asp
BioMedcentral 